\title{
LETTER
}

\section{Intensive care unit acquired muscle weakness in COVID-19 patients}

\author{
Nathalie Van Aerde ${ }^{1}$, Greet Van den Berghe ${ }^{1,3}$, Alexander Wilmer ${ }^{2}$, Rik Gosselink ${ }^{4}$, Greet Hermans , $^{*}$ (I) \\ and COVID-19 Consortium
}

๑ 2020 Springer-Verlag GmbH Germany, part of Springer Nature

\section{Dear Editor,}

Infection with the SARS-CoV-2 virus may lead to hypoxemic respiratory failure and acute respiratory distress syndrome (ARDS). ARDS is frequently complicated by intensive care unit acquired weakness (ICUAW) [1], which is associated with poor outcomes [2]. Critically ill patients affected with coronavirus disease 2019 (COVID19) may differ from typical ARDS-patients in baseline factors [3] and ICU exposures associated with ICUAW [4]. Of particular concern may be the need for deep sedation to avoid patient-ventilator dyssynchrony and ventilator-induced/self-inflicted lung-injury because of high respiratory drive [5]. We aimed to assess the incidence of ICUAW in critically ill COVID-19 patients, to identify factors associated with its occurrence, and to describe its short-term outcomes.

This single-center, retrospective, observational study involved adult critically ill COVID-19 patients admitted to the University Hospitals Leuven, from March 13th until June 8th 2020. After April 1st, physiotherapists were re-engaged in patient care and performed daily strengthassessment when appropriate. Records of eligible patients were searched for baseline characteristics, ICU exposures and outcomes. The primary outcome was the incidence of ICUAW, assessed with the MRC-sum score [2], at awakening, at ICU and hospital discharge in patients

\footnotetext{
*Correspondence: greet.hermans@uzleuven.be

1 Department of Cellular and Molecular Medicine, KU Leuven, Herestraat 49, 3000 Leuven, Belgium

Full author information is available at the end of the article

Greet Van den Berghe, Alexander Wilmer, Rik Gosselink and Greet

Hermans have equally contributed.

The members of the COVID-19 consortium are listed in

"Acknowledgements".
}

requiring invasive mechanical ventilation (IMV). In addition, we evaluated factors and short-term outcomes associated with weakness at ICU discharge. To assess bias, we compared characteristics and outcomes for patients with and without MRC-sum score, and studied patients without IMV.

Of 486 hospitalized COVID-19 patients, 114 required intensive care of whom 74 (64.9\%) needed IMV (Supplemental Fig. 1). Admission and ICU characteristics are provided in the Online Supplement. Total hospital mortality was $60 / 486$ (12.3\%), ICU mortality was $11 / 114$ (9.6\%). All deaths occurred in IMV patients [11/74 (14.9\%)]. In 50/74 (67.6\%) assessed IMV patients, the incidences of ICUAW at awakening, ICU, and hospital discharge were $72 \%, 52 \%$ and $27 \%$ (Fig. 1). Those without MRC-sum-score were older as compared to those with MRC-sum-score [67 (60-76) versus 60 (53-67), $p=0.044]$ and comprised nine patients who died before awakening, possibly introducing selection bias. Admission characteristics were similar between patients with and without ICUAW, but weak patients had prolonged ventilation (days) [24 (15-29) versus $12(8-17), p<0.001$ ], higher mean morning glycemia (mg/dl) [126 (119-134) versus 118 (110-129), $p=0.041$, and more frequently received dialysis $[11 / 26(42.3 \%)$ versus $4 / 24$ (16.7\%), $p=0.048]$. Exposure to corticosteroids, sedatives and analgesics, except for dexmedetomidine, and NMBAs was higher (see Online Supplement). Weak patients had longer ICU stays (days) [30 (19-42) versus 19 (12-25), $p=0.008]$, lower mobility scores at ICU discharge [2 $(2-2)$ versus 6 (4-6), $p<0.001]$, but ICU readmission [0/26 $(0 \%)$ versus $2 / 24(8.3 \%), p=0.225]$ and mortality [2/26 (7.7\%) versus $0 / 24(0 \%), p=0.491]$ were not different. Handgrip-strength (\%pred) [43\% (28-59\%) versus $64 \%(36-80 \%), p=0.045]$, and Barthel at hospital

\section{Springer}



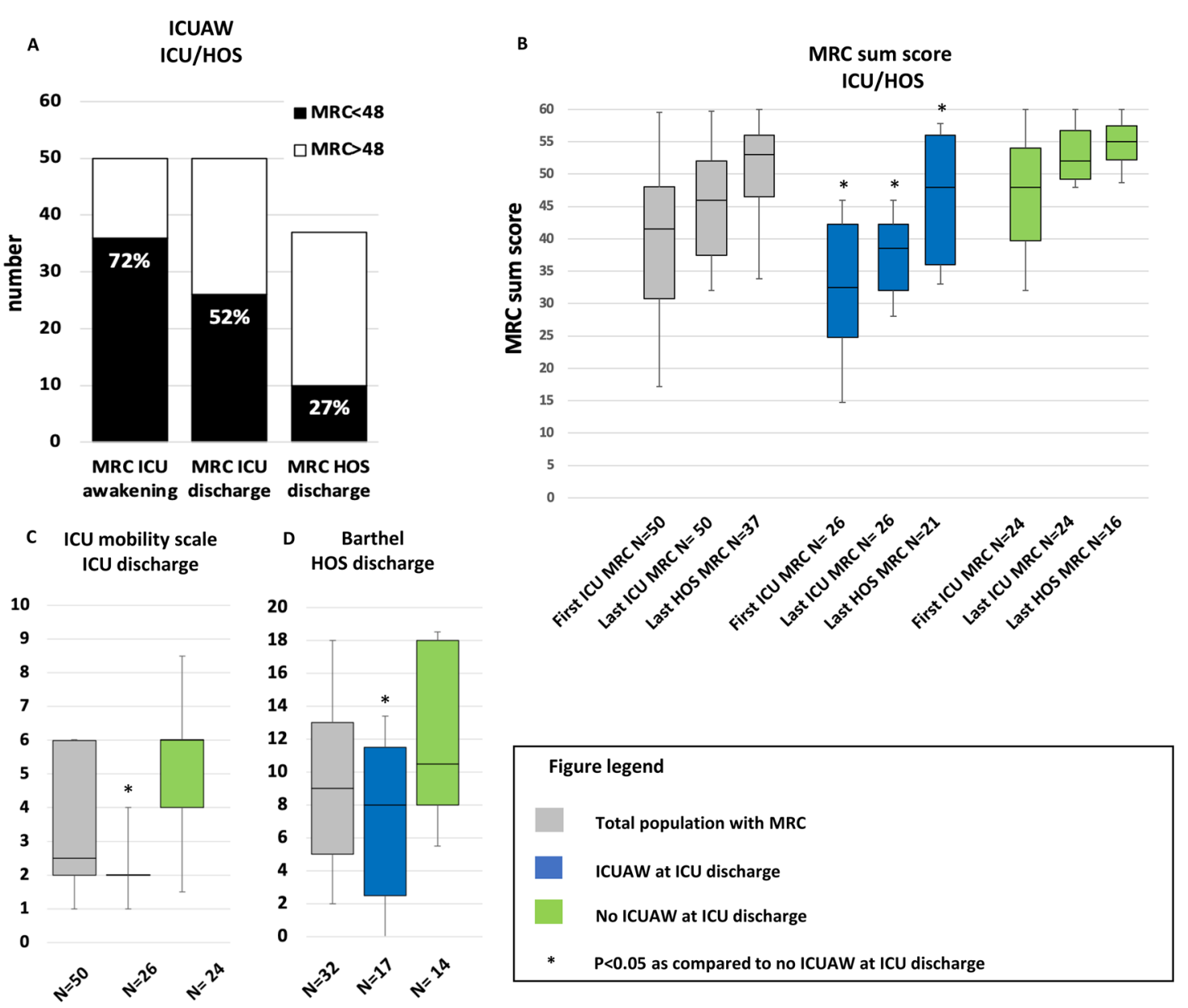

Fig. 1 Strength and functional outcomes in COVID-19 patients, requiring invasive mechanical ventilation, with and without weakness at ICU discharge. Panel A incidence of ICUAW at awakening, ICU discharge and hospital discharge. Panel B MRC-sum-score at awakening, ICU discharge and hospital discharge. Panel C ICU mobility score at ICU discharge. Panel D Barthel score at hospital discharge. MRC Medical Research Council, ICUAW intensive care unit acquired weakness, ICU intensive care unit, HOS hospitalization

discharge [8 (2.5-11.5) versus $10.5(8-18), p=0.040]$, remained lower in weak patients (Fig. 1). 15/26 (57.7\%) weak versus 6/24 (25\%) not-weak patients were referred for in-patient rehabilitation. In 6/40 (15\%) assessed nonIMV patients, one patient was weak at ICU discharge and none at hospital discharge (see Online Supplement).

In conclusion, in this cohort of critically ill COVID19 patients, survival was high, but those needing prolonged sedation frequently presented with ICUAW. Although strength improved throughout hospitalization, impact on functional status remained substantial. These data indicate that there may be a price to pay for allowing rigorous lung-protective ventilation and underscore the need for follow-up of post-ICU COVID-19 patients, to offer tailored rehabilitation, hopefully reducing long-term impact.

\section{Electronic supplementary material}

The online version of this article (https://doi.org/10.1007/s00134-020-06244-7) contains supplementary material, which is available to authorized users.

\section{Author details}

${ }^{1}$ Department of Cellular and Molecular Medicine, KU Leuven, Herestraat 49, 3000 Leuven, Belgium. ${ }^{2}$ Medical Intensive Care Unit, Department of General Internal Medicine, University Hospitals Leuven, Herestraat 49, 3000 Leuven, Belgium. ${ }^{3}$ Department of Intensive Care Medicine, University Hospitals Leuven, Herestraat 49, 3000 Leuven, Belgium. ${ }^{4}$ Department of Rehabilitation Sciences, KU Leuven, Herestraat 49, 3000 Leuven, Belgium.

\section{Acknowledgements}

The members of the COVID-19 Consortium are: Philippe Meersseman: Medical Intensive Care Unit, Department of General Internal Medicine, University Hospitals Leuven, Herestraat 49, 3000, Leuven, Belgium; Jan Gunst: Department of Cellular and Molecular Medicine, KU Leuven, Herestraat 49, 3000, Leuven, Belgium; Department of Intensive Care Medicine, University Hospitals Leuven, Herestraat 49, 3000, Leuven, Belgium; Vicky Aerts: Department of Rehabilitation Sciences, KU Leuven, Herestraat 49, 3000, Leuven, Belgium; Tim Balthazar: Department of Cardiovascular Diseases, University Hospitals Leuven, 
Herestraat 49, 3000, Leuven, Belgium; Astrid Barbé: Department of Anaesthesiology, University Hospitals Leuven, Herestraat 49, 3000, Leuven, Belgium; Arne Böhrer: Department of Rehabilitation Sciences, KU Leuven, Herestraat 49, 3000, Leuven, Belgium; Department of Physical and Rehabilitation Medicine, University Hospitals Leuven, Herestraat 49, 3000, Leuven, Belgium; Kim Caluwé: Department of Rehabilitation Sciences, KU Leuven, Herestraat 49, 3000, Leuven, Belgium; Department of Physical and Rehabilitation Medicine, University Hospitals Leuven, Herestraat 49, 3000, Leuven, Belgium; Michael P. Casaer: Department of Cellular and Molecular Medicine, KU Leuven, Herestraat 49, 3000, Leuven, Belgium; Department of Intensive Care Medicine, University Hospitals Leuven, Herestraat 49, 3000, Leuven, Belgium; Iris Coosemans: Department of Rehabilitation Sciences, KU Leuven, Herestraat 49, 3000, Leuven, Belgium; Department of Physical and Rehabilitation Medicine, University Hospitals Leuven, Herestraat 49, 3000, Leuven, Belgium; Steve Coppens: Department of Anaesthesiology, University Hospitals Leuven, Herestraat 49, 3000, Leuven, Belgium; Dieter Dauwe: Department of Cellular and Molecular Medicine, KU Leuven, Herestraat 49, 3000, Leuven, Belgium; Department of Intensive Care Medicine, University Hospitals Leuven, Herestraat 49, 3000, Leuven, Belgium; Yves Debaveye: Department of Cellular and Molecular Medicine, KU Leuven, Herestraat 49, 3000, Leuven, Belgium; Department of Intensive Care Medicine, University Hospitals Leuven, Herestraat 49, 3000, Leuven, Belgium; Johan De Coster: Department of Anaesthesiology, University Hospitals Leuven, Herestraat 49, 3000, Leuven, Belgium; Paul De Munter: Department of General Internal Medicine, University Hospitals Leuven, Herestraat 49, 3000, Leuven, Belgium; Lot Demuynck: Department of Rehabilitation Sciences, KU Leuven, Herestraat 49, 3000, Leuven, Belgium; Department of Physical and Rehabilitation Medicine, University Hospitals Leuven, Herestraat 49, 3000, Leuven, Belgium; Veerle De Sloovere: Department of Anaesthesiology, University Hospitals Leuven, Herestraat 49, 3000, Leuven, Belgium; Lars Desmet: Department of Intensive Care Medicine, University Hospitals Leuven, Herestraat 49, 3000, Leuven, Belgium; Erwin De Troy: Department of Intensive Care Medicine, University Hospitals Leuven, Herestraat 49, 3000, Leuven, Belgium; Greet De Vlieger: Department of Cellular and Molecular Medicine, KU Leuven, Herestraat 49, 3000, Leuven, Belgium; Department of Intensive Care Medicine, University Hospitals Leuven, Herestraat 49, 3000, Leuven, Belgium; Bregje Frickx: Department of Rehabilitation Sciences, KU Leuven, Herestraat 49, 3000, Leuven, Belgium; Department of Physical and Rehabilitation Medicine, University Hospitals Leuven, Herestraat 49, 3000, Leuven, Belgium; Eline Haghedooren: Department of Rehabilitation Sciences, KU Leuven, Herestraat 49, 3000, Leuven, Belgium; Department of Physical and Rehabilitation Medicine, University Hospitals Leuven, Herestraat 49, 3000, Leuven, Belgium; Renata Haghedooren: Department of Intensive Care Medicine, University Hospitals Leuven, Herestraat 49, 3000, Leuven, Belgium; Liesbet Henckaerts: Department of General Internal Medicine, University Hospitals Leuven, Herestraat 49, 3000, Leuven, Belgium; Arne Heyns; Department of Physical and Rehabilitation Medicine, University Hospitals Leuven, Herestraat 49, 3000, Leuven, Belgium; Miek Hornikx: Department of Rehabilitation Sciences, KU Leuven, Herestraat 49, 3000, Leuven, Belgium; Department of Physical and Rehabilitation Medicine, University Hospitals Leuven, Herestraat 49, 3000, Leuven, Belgium; Catherine Ingels: Department of Cellular and Molecular Medicine, KU Leuven, Herestraat 49, 3000, Leuven, Belgium; Department of Intensive Care Medicine, University Hospitals Leuven, Herestraat 49, 3000, Leuven, Belgium; Bart Jacobs: Department of Intensive Care Medicine, University Hospitals Leuven, Herestraat 49, 3000, Leuven, Belgium; Kristel Janssen: Department of Rehabilitation Sciences, KU Leuven, Herestraat 49, 3000, Leuven, Belgium; Department of Physical and Rehabilitation Medicine, University Hospitals Leuven, Herestraat 49, 3000, Leuven, Belgium; Wouter Meersseman: Medical Intensive Care Unit, Department of General Internal Medicine, University Hospitals Leuven, Herestraat 49, 3000, Leuven, Belgium; Geert Meyfroidt: Department of Cellular and Molecular Medicine, KU Leuven, Herestraat 49, 3000, Leuven, Belgium; Department of Intensive Care Medicine, University Hospitals Leuven, Herestraat 49, 3000, Leuven, Belgium; Jan Muller: Department of Intensive Care Medicine, University Hospitals Leuven, Herestraat 49, 3000, Leuven, Belgium; Arne Neyrinck: Department of Anaesthesiology, University Hospitals Leuven, Herestraat 49, 3000, Leuven, Belgium; Marijke Peetermans: Medical Intensive Care Unit, Department of General Internal Medicine, University Hospitals Leuven, Herestraat 49, 3000, Leuven, Belgium; Steffen Rex: Department of Anaesthesiology,
University Hospitals Leuven, Herestraat 49, 3000, Leuven, Belgium; An Schrijvers: Department of Anaesthesiology, University Hospitals Leuven, Herestraat 49, 3000, Leuven, Belgium; Peter Vanbrabant: Department of General Internal Medicine, University Hospitals Leuven, Herestraat 49, 3000, Leuven, Belgium; Christophe Vandenbriele: Department of Cardiovascular Diseases, University Hospitals Leuven, Herestraat 49, 3000, Leuven, Belgium; Raf Van den Eynde: Department of Anaesthesiology, University Hospitals Leuven, Herestraat 49, 3000, Leuven, Belgium; Marleen van den Hauwe: Department of Rehabilitation Sciences, KU Leuven, Herestraat 49, 3000, Leuven, Belgium; Department of Physical and Rehabilitation Medicine, University Hospitals Leuven, Herestraat 49, 3000, Leuven, Belgium; Marc Van de Velde: Department of Anaesthesiology, University Hospitals Leuven, Herestraat 49, 3000, Leuven, Belgium; Marine Van Hollebeke: Department of Rehabilitation Sciences, KU Leuven, Herestraat 49, 3000, Leuven, Belgium; Eric Van Wijngaerden: Department of General Internal Medicine, University Hospitals Leuven, Herestraat 49, 3000, Leuven, Belgium; Sandra Verelst: Department of Emergency Medicine, University Hospitals Leuven, Herestraat 49, 3000, Leuven, Belgium; Dirk Vlasselaers: Department of Cellular and Molecular Medicine, KU Leuven, Herestraat 49, 3000, Leuven, Belgium; Department of Intensive Care Medicine, University Hospitals Leuven, Herestraat 49, 3000, Leuven, Belgium; Claudia Volker: Department of Rehabilitation Sciences, KU Leuven, Herestraat 49, 3000, Leuven, Belgium; Department of Physical and Rehabilitation Medicine, University Hospitals Leuven, Herestraat 49, 3000, Leuven, Belgium; Bart Vrijsen: Department of Rehabilitation Sciences, KU Leuven, Herestraat 49, 3000, Leuven, Belgium; Department of Physical and Rehabilitation Medicine, University Hospitals Leuven, Herestraat 49, 3000, Leuven, Belgium; Joost Wauters: Medical Intensive Care Unit, Department of General Internal Medicine, University Hospitals Leuven, Herestraat 49, 3000, Leuven, Belgium; Pieter J. Wouters: Department of Cellular and Molecular Medicine, KU Leuven, Herestraat 49, 3000, Leuven, Belgium; Department of Intensive Care Medicine, University Hospitals Leuven, Herestraat 49, 3000, Leuven, Belgium.

\section{Compliance with ethical standards}

\section{Conflicts of interest}

The authors declare that they have no conflict of interest.

\section{Publisher's Note}

Springer Nature remains neutral with regard to jurisdictional claims in published maps and institutional affiliations.

Accepted: 7 September 2020

Published online: 28 September 2020

\section{References}

1. Herridge MS, Tansey CM, Matté A, Tomlinson G, Diaz-Granados N, Cooper A et al (2011) Functional disability 5 years after acute respiratory distress syndrome. N Engl J Med 364(14):1293-1304

2. Hermans $G$, Van Mechelen H, Clerckx B, Vanhullebusch T, Mesotten D, Wilmer A et al (2014) Acute outcomes and 1-year mortality of intensive care unit-acquired weakness. A cohort study and propensity-matched analysis. Am J Respir Crit Care Med 190(4):410-420

3. Grasselli G, Zangrillo A, Zanella A, Antonelli M, Cabrini L, Castelli A et al (2020) Baseline characteristics and outcomes of 1591 patients infected with SARS-CoV-2 admitted to ICUs of the Lombardy Region, Italy. JAMA 323(16):1574-1581

4. Alhazzani W, Møller MH, Arabi YM, Loeb M, Gong MN, Fan E et al (2020) Surviving Sepsis Campaign: guidelines on the management of critically ill adults with Coronavirus Disease 2019 (COVID-19). Intensive Care Med 46:854-887. https://doi.org/10.1007/s00134-020-06022-5

5. Kapp CM, Zaeh S, Niedermeyer S, Punjabi NM, Siddharthan T, Damarla M (2020) The use of analgesia and sedation in mechanically ventilated patients with COVID-19 ARDS. Anesth Analg. https://doi.org/10.1213/ ANE.0000000000005131 